\title{
Diseño e implementación de un sistema seguidor solar inteligente a dos ejes para optimizar la producción de energía fotovoltaica con una lente de fresnel y un sistema de limpieza robotizado a bajo costo
}

\section{Design and implementation of an intelligent solar follower system of two axes to optimize the production of photovoltaic energy with a fresnel lens and a robotized low cost cleaning system}

\author{
HERNÁNDEZ-MEDINA，José Juan†*，LÓPEZ-XELO，Hilario，HERNÁNDEZ-DÍAZ，Aldo y \\ CABRERA-PÉREZ, José Luis
}

Instituto Tecnológico Superior de Tlaxco

ID $1^{\text {er }}$ Autor: José Juan, Hernández-Medina / ORC ID: 0000-0003-4625-2660, CVU CONACYT ID: 5227576

ID 1 ${ }^{\text {er }}$ Coautor: Hilario, López-Xelo / ORC ID: 0000-0001-6597-6598, CVU CONACYT ID: 732365

ID $2^{\text {do }}$ Coautor: Aldo, Hernández-Díaz / ORC ID: 0000-0002-5338-8075, CVU CONACYT ID: 269589

ID $3^{\text {er }}$ Coautor: José Luis, Cabrera-Pérez / ORC ID: 0000-0003-3035-0266, CVU CONACYT ID: 672263

DOI: $10.35429 /$ JRE.2019.11.3.1.6

Recibido 16 de Octubre, 2019; Aceptado 19 Noviembre, 2019

\section{Resumen}

Los sistemas fotovoltaicos con lámparas de Fresnel son costosos, así como tecnológicamente complicados, debido a sus componentes óptico, mecánico y de control. Todos estos sistemas son sensibles a los costos de operación y mantenimiento. En este artículo se propone utilizar lentes de Fresnel comerciales para mejorar la eficiencia de los módulos fotovoltaicos, en conjunto con un sistema seguidor solar inteligente de control difuso a dos ejes y un novedoso sistema de limpieza robotizado de bajo costo. La idea básica es que este sistema optimice la producción de energía eléctrica de manera económica y tecnológicamente simple. Se recurrirá a la tecnología de microcontroladores Arduino y la lógica difusa para el control, así como la geometría de las lentes de Fresnel, para concentrar en un área pequeña la energía solar, sin hablar de que tienen estos dispositivos ópticos tienen un peso reducido. El efecto acumulado de producción de energía se verá mejorado por el sistema robotizado de limpieza de bajo costo. Se debe mantener la mayor parte del tiempo posible la alineación y perpendicularidad del impacto de la energía solar sobre las celdas fotovoltaicas y contrastar el desempeño de este sistema con otros comerciales, más caros y tecnológicamente más complejos.

Fotovoltaico, Lentes de Fresnel, Control difuso

\begin{abstract}
Photovoltaic systems with Fresnel lenses are expensive, as well as technologically complex, due to their optical, mechanical and control components. In addition, all these systems are sensitive to operation and maintenance costs. This article proposes the use of commercial Fresnel lenses to improve the efficiency of photovoltaic modules, in conjunction with a fuzzy intelligent controlled solar tracking system with two-axis and a novel low-cost robotic cleaning system. The basic idea is that this system optimizes the production of electricity in an economically and technologically simple way. The technology of Arduino microcontrollers and fuzzy logic for control, as well as the geometry of Fresnel lenses, will be used to concentrate solar energy in a small area, not to mention that these optical devices have a low weight. The accumulated effect of energy production will be improved by a novel low cost robotic cleaning system. The alignment and perpendicularity of the impact of solar energy on photovoltaic cells must be maintained as much as possible and the performance of this system must be compared with other more expensive and technologically more complex commercial systems.
\end{abstract}

Photovoltaic, Fresnel lens, fuzzy control

Citación: HERNÁNDEZ-MEDINA, José Juan, LÓPEZ-XELO, Hilario, HERNÁNDEZ-DÍAZ, Aldo y CABRERA-PÉREZ, José Luis. Diseño e implementación de un sistema seguidor solar inteligente a dos ejes para optimizar la producción de energía fotovoltaica con una lente de fresnel y un sistema de limpieza robotizado a bajo costo. Revista de Energías Renovables 2019. 3-11: $1-6$

\footnotetext{
*Correspondencia al Autor (Correo Electrónico: jjhmedina@gmail.com)

$\dagger$ Investigador contribuyendo como primer autor.
} 


\section{Introducción}

Las tecnologías de concentración de energía solar, CPV, por sus siglas en inglés (Concentrating Photovoltaic), mediante lentes de Fresnel es una forma efectiva de optimizar la incidencia de la luz solar en las fotoceldas ya que los sistemas CPV, pueden contribuir a reducir el área de las celdas fotovoltaicas y puede permitir la reducción de costos en general del sistema (Yupeng et al, 2012).

Los concentradores funcionan mejor cuando hay una fuente de luz directa y el concentrador puede apuntar directamente a ella. Se debe recordar que las celdas fotovoltaicas (FV) tienen una baja eficiencia: los paneles monocristalinos tienen un promedio de $16 \%$ y los paneles y los paneles amorfos apenas superan el $10 \%$.

Para los satélites y las naves espaciales se fabrican módulos de arseniuro de galio (GaAs) que alcanzan una eficiencia de 30\% (Shanks, 2016).

El propósito de este proyecto es mejorar la eficiencia de las fotoceldas con materiales baratos como las lentes plásticas concentradoras de Fresnel, para mejorar la producción de energía eléctrica, reduciendo el área útil.

Las lentes de Fresnel para este concentrador solar se deben acoplar a elementos secundarios ópticos (SOE o secondary optical elements, por sus siglas en inglés), para incrementar la concentración geométrica de los haces de luz, así como para ajustar la tolerancia del ángulo, reducir las pérdidas de eficiencia por calentamiento excesivo y a otros dispositivos para controlar la posición con respecto al sol (Vallerotto et al, 2016).

\section{Metodología}

La parte medular de este sistema es un controlador inteligente difuso, que permite orientar los paneles FV, a fin de mantener siempre la perpendicularidad del panel con respecto al Sol.

El seguidor inteligente tendrá dos ejes, uno de cuales permite seguir al sol en su recorrido diario aparente de oriente a poniente y el segundo eje es para seguir al sol a lo largo de su recorrido estacional.
La parte mecánica del sistema comprende las lentes de Fresnel, el sistema robotizado de limpieza, el seguidor solar, un microcontrolador, la estructura de montaje, un SPCC (Solar Power Charge Controller) o controlador de carga por energía solar, las baterías, motores paso a paso y el sensor para detectar la luz. Dado que el sistema es un seguidor inteligente tomará las decisiones pertinentes, incluso en condiciones nubosas, lluviosas e imprevistas como un eclipse (Huang et al, 2016).

\section{Mercado Objetivo del proyecto}

En México hay más de 2,200,000 personas sin servicios de energía eléctrica procedente de una red (INEGI, 2010), que viven en regiones apartadas y que son los principales clientes potenciales para este proyecto.

Por otra parte, este sistema debe funcionar durante al menos 10 años, sin gastos mayores en mantenimiento, pues sólo requerirá lubricación e inspecciones periódicas. Además, el sistema debe soportar la intemperie, sobre todo la lluvia y el granizo, así como el viento y podría operar en rangos de temperatura de entre $-15^{\circ} \mathrm{C}$ a $50^{\circ} \mathrm{C}$. El Protocolo de Kioto establece que para 2050 habrá una matriz energética libre de combustibles fósiles, con tres pilares: el ahorro de energía, el fomento de las fuentes renovables y la eficiencia energética (Font, 2019).

Además, otras aplicaciones que pueden usar este sistema son los sistemas autónomos de bombeo de agua y de comunicaciones, los sistemas de protección catódica de metales expuestos a la intemperie o a la humedad, luces indicadoras para tráfico aéreo y ferroviario, suministrar energía a naves, entre otras. Además, como ya se hace en muchos lugares, la producción de energía en paneles solares, reducirá la demanda de electricidad del sector convencional (Kumar et al, 2015).

\section{El control inteligente difuso}

La lógica difusa es un algoritmo creado por Lofty Zadeh en 1965 y consiste en una teoría general de conjuntos, en la que cada elemento tiene un número infinito de grados de pertenencia a uno o más conjuntos difusos cuyos valores de pertenencia oscilan entre cero y uno. 
En contraste, en los conjuntos tradicionales (también llamados booleanos o duros), los elementos pertenecen o no pertenecen a dicho conjunto, asumiendo solamente dos valores: cero o uno (verdadero o falso). (Huang et al, 2016).

Mediante la lógica difusa se pueden diseñar controladores inteligentes difusos o FLC, por sus siglas en inglés (Fuzzy Logic Controllers), capaces de tratar con situaciones ambiguas, complejas y situaciones en las que se tiene como entrada información fragmentaria.

El controlador inteligente difuso se basa en algunas reglas difusas. Las reglas difusas representan el compendio del conocimiento de un experto humano en el sistema que se desea controlar (Patel y Shewale, 2015). El criterio del experto se expresa en términos de reglas empíricas arbitrarias del tipo si... entonces... Dichas reglas determinan las interacciones entre las variables difusas, por ejemplo:

\section{Si está muy oscuro entonces enciende} todas las luces.

El FLC se compone de cuatro etapas: la fuzzificación, la base de reglas, el mecanismo de inferencia y la defuzzificación (Usta et al, 2011). Cada variable que interviene se representa mediante un conjunto difuso.

\section{Etapa 1: La fuzzificación}

Para realizar esta etapa se debe definir en primer término, un universo de discurso que permita representar de manera adecuada los valores de las variables de entrada. Después, hay que convertir los valores numéricos de dicha entrada en términos que permitan definir los grados de pertenencia de los miembros de los conjuntos difusos, a través de una función de pertenencia (Aguirre et al, 2018). Como ya se mencionó, dichos valores de pertenencia oscilan entre 0 y 1 , para realizar una representación de estas señales versus el voltaje del panel FV para diferentes niveles de irradiación solar (Robles y Hómez, 2011)

Los nombres de las variables de entrada son arbitrarios $\mathrm{y}$ en este sistema son la iluminación en la mañana (AM) y la iluminación en la tarde (PM), variables que representan la cantidad de luz recibida en la mañana y en la tarde por el módulo FV.
A su vez, la variable AM (iluminación por la mañana), se dividió en los siguientes conjuntos difusos arbitrarios: muy_débil, débil, media, fuerte, muy_fuerte.

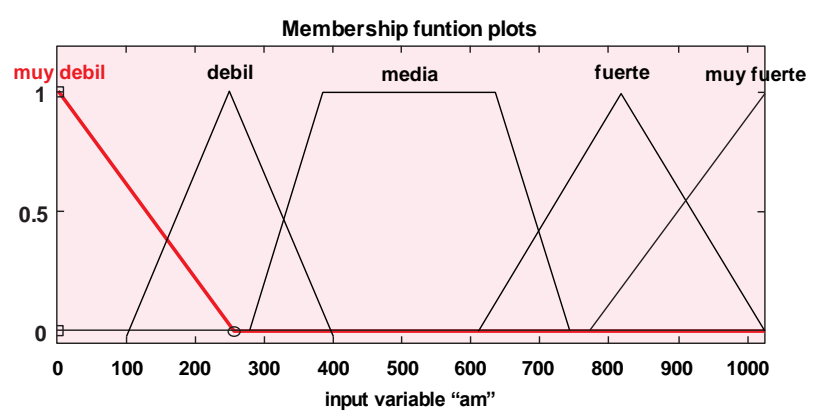

Gráfico 1 La variable de entrada AM (iluminación por la mañana) que tiene cinco conjuntos difusos: muy_débil, débil, media, fuerte, muy_fuerte

En cuanto a la variable PM se dividió en los siguientes conjuntos difusos arbitrarios: muy_débil, débil, media, fuerte, muy_fuerte, como lo muestra la siguiente figura.

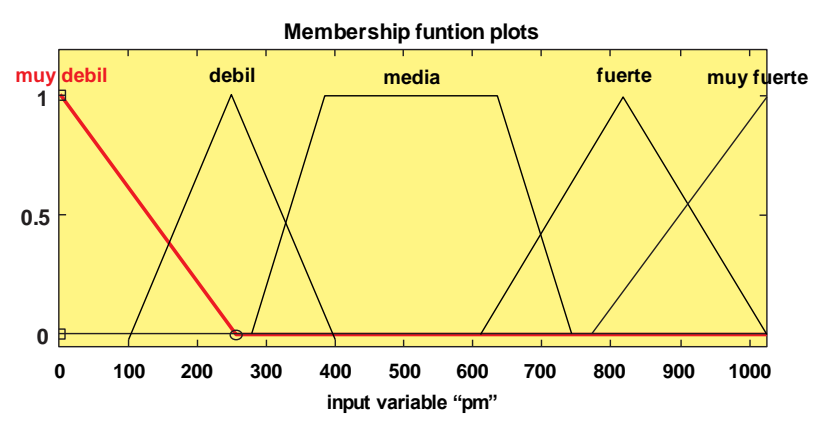

Gráfico 2 La variable de salida PM (iluminación por la tarde) con cinco conjuntos difusos: muy_débil, débil, media, fuerte, muy_fuerte

La variable de salida, tiene el nombre de colocación y representa la posición del panel FV y tiene cinco conjuntos: ext_oriente, oriente, medio, poniente, ext_poniente.

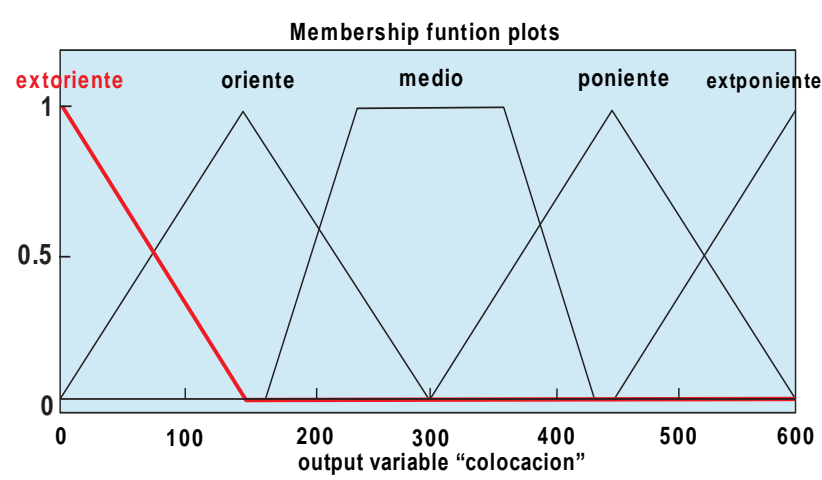

Gráfico 3 La variable de salida COLOCACIÓN representa la orientación del panel con cinco conjuntos difusos: ext_oriente, oriente, medio, poniente, ext_poniente 


\section{Etapa 2: La base de reglas difusas}

Las 25 reglas difusas de control de este sistema se evalúan mediante un mecanismo de inferencia y se representan como un conjunto de silogismos del tipo SI... ENTONCES..., mismos que se pueden resumir en la Tabla 1

\begin{tabular}{|l|l|l|l|l|l|}
\hline PM AM & \multicolumn{1}{|c|}{$\begin{array}{l}\text { Muy } \\
\text { débil }\end{array}$} & \multicolumn{1}{|c|}{ Débil } & \multicolumn{1}{|c|}{ Media } & \multicolumn{1}{|c|}{ Fuerte } & \multicolumn{1}{c|}{$\begin{array}{c}\text { Muy } \\
\text { Fuerte }\end{array}$} \\
\hline $\begin{array}{l}\text { Muy } \\
\text { débil }\end{array}$ & Medio & Oriente & $\begin{array}{l}\text { Ext_ } \\
\text { oriente }\end{array}$ & $\begin{array}{l}\text { Ext_ } \\
\text { oriente }\end{array}$ & $\begin{array}{l}\text { Ext_ } \\
\text { oriente }\end{array}$ \\
\hline Débil & Poniente & Medio & Oriente & $\begin{array}{l}\text { Ext_ } \\
\text { oriente }\end{array}$ & $\begin{array}{l}\text { Ext_ } \\
\text { oriente }\end{array}$ \\
\hline Media & Poniente & poniente & Medio & oriente & Oriente \\
Fuerte & $\begin{array}{l}\text { Ext_- } \\
\text { poniente }\end{array}$ & $\begin{array}{l}\text { Ext_- } \\
\text { poniente }\end{array}$ & poniente & medio & Oriente \\
$\begin{array}{l}\text { Muy } \\
\text { Fuerte }\end{array}$ & $\begin{array}{l}\text { Ext_- } \\
\text { poniente }\end{array}$ & $\begin{array}{l}\text { Ext_- } \\
\text { poniente }\end{array}$ & $\begin{array}{l}\text { Ext_- } \\
\text { poniente }\end{array}$ & poniente & Medio \\
\hline
\end{tabular}

Tabla 1 Las reglas de inferencia del seguidor solar

Un ejemplo sobre cómo se debe interpretar la tabla anterior, tome por caso el recuadro verde como base, mismo que se puede leer por sus intersecciones en sus ejes:

\section{Si AM es media y PM es débil, entonces el panel se debe orientar hacia el oriente}

A partir de los valores de entrada y mediante el uso de los conjuntos difusos, se pueden cuantificar las funciones de pertenencia.

Las variables difusas establecen la relación entre la luz solar recibida y la orientación que debería tener el panel fotovoltaico, que a su vez es función de la fuerza electromotriz producida en el panel FV (Morales y Espinosa, 2018).

\section{Etapa 3: La inferencia difusa}

El motor de inferencia difusa es la "mente" del sistema experto y es el que toma las decisiones al interpretar y aplicar el conocimiento experto empírico a partir de reglas difusas.

Cada regla difusa se evalúa de izquierda a derecha, pero todas ellas son tratadas como si fueran evaluadas simultáneamente. La operación difusa and se usa para conectar los antecedentes con los consecuentes dentro de una misma regla.

El tratamiento de las reglas sucesivas se realiza mediante la operación difusa or. A este procedimiento difuso, AND - OR se le llama razonamiento de tipo Mamdani.

\section{Etapa 4: La defuzzificación}

La defuzzificación es el proceso de interpretar los grados de pertenencia de los conjuntos difusos de salida. Las funciones de pertenencia de salida tienen forma de triángulo. Si este triángulo fuera cortado por una línea horizontal en un punto entre la parte superior e inferior, y la parte superior fuese eliminada, la porción restante sería un trapezoide. Todos los trapezoides así formados se superponen uno sobre otro formarían una única figura geométrica compleja. Luego, se calcula el centroide de esta figura y se obtiene un valor duro, mismo que corresponde a una decisión concreta. En otras palabras, la etapa de defuzzificación permite que las decisiones del mecanismo de inferencia se concreten en términos de salidas duras, es decir, encendido o apagado (Patel y Shewale, 2015).

\section{La protección catódica del sistema}

Los paneles solares deben tener una unidad catódica de protección (CP por sus siglas en inglés, cathodic protección unit). La CP es un convertidor de corriente que suministra una CD a las tuberías, herrajes, tornillería y los otros equipos metálicos expuestos a la intemperie para mitigar los efectos de la corrosión. A fin de disminuir el mantenimiento la CP se fabrica de elementos de estado sólido.

\section{El concentrador solar}

Las lentes de Fresnel para concentradores solares son fabricados con vidrio-silicón (SOG, por sus siglas en inglés silicon on glass), ya que es el material que tiene el mejor desempeño y son adecuados para operar a la intemperie, además de tener las siguientes ventajas:

- $\quad$ Alto índice de refracción.

- $\quad$ Pérdidas ópticas mínimas

- Transmitancia de alta intensidad.

- $\quad$ Mayor resistencia a la radiación UV y al envejecimiento.

- $\quad$ Las lentes SOG son varias veces mayor resistencia a la intemperie que el PMMA (polimetil meta acrilato óptico).

Además, la superficie de la lente de Fresnel es ultrablanca y endurecida, lo que resulta que los surcos de la lente están perfectamente definidos para optimizar el proceso de concentración. 
Los fabricantes de lentes de Fresnel pueden hacer algunas modificaciones para adaptar su producto a las necesidades del cliente, en términos de sus ángulos de incidencia, de la distancia focal entre el lente y el panel solar, entre otros.

La distancia focal de las lentes de Fresnel comerciales oscila entre los $48 \mathrm{~mm}$ y los 350 $\mathrm{mm}$. Los paneles solares más grandes tienen de dimensiones de longitud hasta $1100 \mathrm{~mm}$. En cualquier caso, se sugiere consultar las especificaciones del fabricante de las lentes.

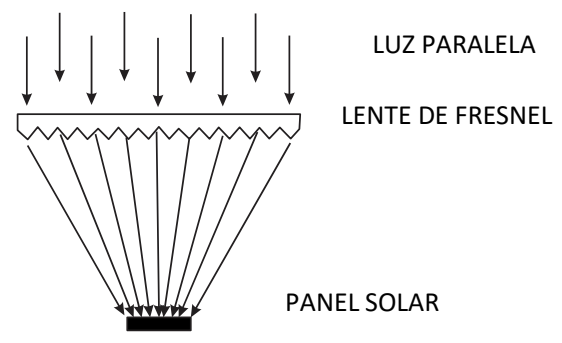

Figura 1 Se muestra cómo los rayos del sol al incidir sobre la lente de Fresnel se concentran de la manera más homogénea posible sobre la superficie del panel solar

\section{El sistema robotizado de limpieza}

El polvo y los contaminantes acumulados sobre la superficie del panel solar bloquean la irradiación solar y en consecuencia la eficiencia puede disminuir considerablemente. Por lo tanto, la limpieza de los paneles solares es de gran interés para garantizar la producción eficiente de la energía. Entre los métodos más usados para la limpieza están los siguientes:

$\begin{array}{ll}- & \text { Cepillado. } \\ - & \text { Sistemas electrostáticos. } \\ - & \text { Lavado con agua. } \\ - & \text { Método con tensoactivos (surfactantes). } \\ - & \text { Sistema de Micro-estructura repelente al } \\ & \text { agua. } \\ - & \text { Sistema de limpieza por ultrasonido. } \\ - & \text { Método de inclinación (Tilt). }\end{array}$

El sistema de limpieza robotizado consiste en un brazo rotatorio con un rociador de agua. Dicho fluido no solamente permitirá realizar la limpieza, sino que también permitirá enfriar los paneles. Este robot para limpieza de desplazamiento cartesiano estará alimentado por su propia fotocelda. El sistema de limpieza contará con un sensor de temperatura que activará a este robot cuando la temperatura se aproxime a los $45^{\circ} \mathrm{C}$, cuidando siempre del choque térmico que pudiese afectar a las fotoceldas.

\section{Resultados}

La posición del sol se monitorea continuamente mediante sensores tipo fotorresistor para conocer las entradas (AM y PM). El voltaje producido en cada sensor de entrada se convierte a un valor digital usando un convertidor analógico a digital (ADC, por sus siglas en inglés, analog digital conversor). Cuando el sistema toma la decisión respecto al ángulo al que se debe colocar el panel, un motor paso a paso hace girar al panel solar de tal manera que se coloque en una posición perpendicular al sol. Los resultados de la simulación en Matlab se muestra en la gráfica número 5 , en la que si se proporciona dos entradas (conjuntos difusos en amarillo, se puede obtener una salida, el conjunto difuso colocación). El control responde en tiempo real.

El motor paso a paso, mismo que impulsa al seguidor solar no funciona todo el tiempo, sino que lo hace cada cinco minutos durante doce horas, es decir, el motor de pasos opera 144 veces en un día, lo que permite ahorrar energía y posicionar las celdas de manera adecuada. Otra rutina de programación permite que cuando llega la noche, el panel solar se coloque de tal manera que regrese a su punto de origen para esperar la salida del sol con un solo arranque del motor. El controlador inteligente difuso toma las mejores decisiones para posicionar el panel, incluso si el día está nublado, lluvioso, o si ocurriera un evento inesperado como un eclipse. Aunque el microcontrolador tiene notables ventajas como su bajo precio y su facilidad para programarse, puede presentar algunos problemas al tratar con sistemas de control complejos.

\section{Conclusiones}

Las fotoceldas son dispositivos que permiten obtener energía eléctrica rentable, si se operan con un criterio de optimización tecnológica. Con tal finalidad, en este trabajo se propone la sinergia de tres elementos importantes: el control inteligente difuso para el seguidor solar, la lente de Fresnel, además de un sistema robotizado para limpieza y enfriamiento de todo el equipo. En suma, el desempeño de los paneles fotovoltaicos se puede optimizar mediante la adición de dispositivos secundarios baratos. Además, es importante que estos dispositivos auxiliares tengan sus propias fotoceldas, independientes para no incurrir en el consumo de la energía del mismo sistema.

HERNÁNDEZ-MEDINA, José Juan, LÓPEZ-XELO, Hilario, HERNÁNDEZ-DÍAZ, Aldo y CABRERA-PÉREZ, José Luis. Diseño e implementación de un sistema seguidor solar inteligente a dos ejes para optimizar la producción de energía fotovoltaica con una lente de fresnel y un optimizar la producción de energía fotovoltaica con una lente de fresnel y un
sistema de limpieza robotizado a bajo costo. Revista de Energías Renovables 2019. 
Por otra parte, también se trata de diseñar este sistema con los medios fácilmente disponibles para su fabricación y mantenimiento y adaptándolo al entorno. La idea es tener una fuente de energía confiable y de calidad. El control difuso parece que tiene un desempeño eficiente en tiempo real, por lo menos en simulación. Un trabajo a futuro es tratar este problema con algún recurso de cómputo paralelo como FPGA o una tarjeta NVIDIA (Patel y Shewale, 2015). Otra perspectiva es interconectar este sistema con una red inteligente (smart grid) para producir un intercambio de energía, permitiendo una mejor gestión de este recurso. Esto puede cambiar el paradigma de generación centralizada de energía, basada en consumidores pasivos y permitiría al usuario del servicio contaminar menos, gestionar mejor su energía y ahorrar dinero (Rubio, 2019).

\section{Referencias}

Abouobaida, H., \& Said, E. B. (2017). Practical Performance Evaluation of Maximum Power Point Tracking Algorithms in a Photovoltaic System. International Journal of Power Electronics and Drive Systems (IJPEDS), 8(4), 1744-1755.

Aguirre, J. C. P., Agundis, A. R., Segura, E. J. R., \& Nolasco, J. J. M. (2018). Control Difuso Para Un Convertidor Cd-Cd Aplicado A Sistemas Fotovoltaicos En Los Modos MPPT y CV. Pistas Educativas, 39(128).

El-Sayed, M. A., \& Leeb, S. (2015, April). Fuzzy logic based maximum power point tracking using boost converter for solar photovoltaic system in Kuwait. In International conference on renewable energies and power quality, Spain (Vol. 14, No. 13, pp. 433-443).

Font, J. G. (2019). La integración de medidas de eficiencia energética en el sector de la edificación en España a la vista de los objetivos de la UE para los horizontes 2020-2030. El district heating and cooling. Revista Catalana de Dret Ambiental, 10(1).

Huang, C. H., Pan, H. Y., \& Lin, K. C. (2016). Development of intelligent fuzzy controller for a two-axis solar tracking system. Applied Sciences, 6(5), 130.

Kharb, R. K., Shimi, S. L., Chatterji, S., \& Ansari, M. F. (2014). Modeling of solar PV module and maximum power point
Kumar, A. P., Parimi, A. M., \& Rao, K. U. (2015, February). Implementation of MPPT control using fuzzy logic in solar-wind hybrid power system. In Signal Processing, Informatics, Communication and Energy Systems (SPICES), 2015 IEEE International Conference on (pp. 1-5). IEEE.

Lee, C. Y., Chou, P. C., Chiang, C. M., \& Lin, C. F. (2009). Sun tracking systems: a review. Sensors, 9(5), 3875-3890.

Morales Ospina, D. A., \& Espinosa Romero, F. A. (2018). Lámpara Led Inteligente Alimentada Por Paneles Solares.

Patel, R. R., \& Shewale, M. A. N. (2015). Intelligent Sun Tracking System Using FLC Implemented On FPGA. Int. J. Adv. Found. Res. Comput, 2(10), 260-263.

Robles, C., \& Hómez, G. A. V. (2011). Control del punto de máxima potencia de un panel solar fotovoltaico, utilizando lógica difusa. Télématique: Revista Electrónica de Estudios Telemáticos, 10(2), 54-72.

Rubio del Castillo, I. (2019). Diseño de un sistema inteligente de gestión energética para hogar con autogeneración y V2G para la integración de smart grids basado en Raspberry Pi.

Shanks, K., Senthilarasu, S., \& Mallick, T. K. (2016). Optics for concentrating photovoltaics: Trends, limits and opportunities for materials and design. Renewable and Sustainable Energy Reviews, 60, 394-407.

Suganthi, L., Iniyan, S., \& Samuel, A. A. (2015). Applications of fuzzy logic in renewable energy systems-a review. Renewable and Sustainable Energy Reviews, 48, 585-607.

Usta, M. A., Akyazi, Ö., \& Altaş, İ. H. (2011, December). Design and performance of solar tracking system with fuzzy logic controller used different membership functions. In Electrical and Electronics Engineering (ELECO), 2011 7th International Conference on (pp. II-381). IEEE.

Vallerotto, G., Victoria, M., Askins, S., Herrero, R., Domínguez, C., Antón, I., \& Sala, G. (2016). Design and modeling of a cost-effective achromatic Fresnel lens for concentrating photovoltaics. Optics express, 24(18), A1245A1256.

Zadeh, L.A. Fuzzy sets. Inf. Control 1965, 8, 338-353. [CrossRef]

HERNÁNDEZ-MEDINA, José Juan, LÓPEZ-XELO, Hilario, HERNÁNDEZ-DÍAZ, Aldo y CABRERA-PÉREZ, José Luis. Diseño e implementación de un sistema seguidor solar inteligente a dos ejes para optimizar la producción de energía fotovoltaica con una lente de fresnel y un sistema de limpieza robotizado a bajo costo. Revista de Energías Renovables 2019. 